\title{
Width-to-length ratio comparison between ameloblastomas and odontogenic keratocysts in the body of the mandible: A preliminary study
}

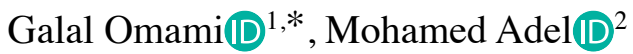 \\ ${ }^{1}$ Department of Oral Health Practice, University of Kentucky College of Dentistry, Lexington, KY, USA \\ ${ }^{2}$ Department of Oral Health Science, University of Kentucky College of Dentistry, Lexington, KY, USA
}

\section{ABSTRACT}

Purpose: The purpose of this study was to investigate the utility of the width-to-length ratio for the differentiation of ameloblastomas and odontogenic keratocysts in the body of the mandible.

Materials and Methods: This study retrospectively reviewed 9 patients with ameloblastomas and 9 patients with odontogenic keratocysts using cone-beam computed tomography. The width-to-length ratio was determined by measuring the ratio between the greatest buccolingual dimension and the greatest perpendicular anteroposterior dimension of the lesion on the axial view. One-way analysis of variance was used to examine the difference in the width-to-length ratio between the 2 types of lesions. Statistical significance was tested at $P<0.05$.

Results: Ameloblastomas showed a mean width-to-length ratio of 0.64 , whereas odontogenic keratocysts showed a mean width-to-length ratio of 0.41 . The cut-off value with which the 2 types of lesions were differentiated was 0.5 . The width-to-length ratios of ameloblastomas were significantly higher than those of odontogenic keratocysts $(P<0.05)$.

Conclusion: The width-to-length ratio might be used to differentiate between ameloblastomas and odontogenic keratocysts. (Imaging Sci Dent 2020; 50: 319-22)

KEY WORDS: Odontogenic Tumors; Mandibular Neoplasms; Mandible; Cone-Beam Computed Tomography

\section{Introduction}

Ameloblastomas and odontogenic keratocysts are the most common odontogenic lesions of the jaw, which are characterized by aggressive local growth and a high recurrence rate. Ameloblastomas account for $10 \%$ of all odontogenic tumors and $1 \%$ of all oral tumors. ${ }^{1}$ Odontogenic keratocysts (also known as keratocystic odontogenic tumors) represent approximately $12 \%$ of all jaw cysts. ${ }^{2}$ The 2 lesions share many radiologic features and often are indistinguishable on diagnostic images. Although odontogenic keratocysts tend to grow along the bone, without the same amount of expansion seen with ameloblastomas, there may

Received June 7, 2020; Revised August 15, 2020; Accepted August 18, 2020 *Correspondence to : Prof. Galal Omami

Department of Oral Health Practice, University of Kentucky College of Dentistry, 800 Rose Street, Lexington, KY 40536, USA

Tel) 1-859-323-8901,E-mail) Galal.Omami@uky.edu still be considerable expansion when keratocysts achieve a large size. However, the difference in bone expansion can be used as a radiographic finding to differentiate between ameloblastomas and odontogenic keratocysts. ${ }^{3}$

Previous studies indicated that some radiographic findings are particularly helpful for the diagnosis of ameloblastomas and odontogenic keratocysts, such as buccolingual expansion, the number of locules, internal density, and root resorption of the adjacent teeth. ${ }^{4-7}$ However, to the best of our knowledge, no study has ever attempted to analyze the width (buccolingual expansion) of lesions in relation to their length (anteroposterior dimension) in this context.

The aim of this preliminary study was to retrospectively investigate the feasibility of the width-to-length ratio for differentiating between ameloblastomas and odontogenic keratocysts in the body of the mandible using cone-beam computed tomography (CBCT). 


\section{Materials and Methods}

CBCT imaging data of patients with conventional ameloblastomas or odontogenic keratocysts of the mandible between 2010 and 2019 were retrospectively retrieved from the picture archiving and communication system of the University of Kentucky College of Dentistry.

The inclusion criteria included: 1) complete clinical records, 2) histological confirmation of the lesion as conventional ameloblastoma or odontogenic keratocyst, and 3) availability of high-quality images without motion artifacts. Exclusion criteria included; 1) lesions in the mandibular ramus, 2) recurrent lesions in the mandible, and 3) the presence of nevoid basal cell carcinoma syndrome.

Nine ameloblastomas ( 6 males and 3 females; median age 38 years; range 10-81 years) and 9 odontogenic keratocysts ( 7 males and 2 females; median age 24 years; range $10-59$ years) were included in this study.

All images were displayed on a 27-inch HD monitor with a screen resolution of $1920 \times 1080$ pixels (Dell P2717H; Dell Inc., Round Rock, TX, USA), and were viewed by a board-certified oral and maxillofacial radiologist. Images were observed under free angulation of three-dimensional (3D) slices in the multiplanar reconstruction module in InVivo dental software (Anatomage Inc., San Jose, CA, USA). Quantitative measurements were recorded twice by a single examiner at an interval of 2 weeks, and the mean values were used for analysis. The new concept of the width-to-length ratio was applied to evaluate the expansion rate of the lesions. The width-to-length ratio was determined by measuring the ratio between the greatest width of the lesion and the greatest length perpendicular to this width on the axial view (Figs. 1 and 2).

The initial statistical approach was to calculate descriptive statistics of the collected data for the width-to-length ratio of both groups of lesions and to test the normality of the data. The significance of the difference in the width-tolength ratio between the 2 types of lesions was tested with one-way analysis of variance. The data were analyzed with JMP Software (JMP, SAS Institute Inc., Cary, NC, USA). Statistical significance was set at $P<0.05$.

\section{Results}

Ameloblastomas showed a mean width-to-length ratio of 0.64 , whereas odontogenic keratocysts showed a mean width-to-length ratio of 0.41 (Fig. 3). The width-to-length ratio of ameloblastomas was significantly higher than that of odontogenic keratocysts $(P<0.05$, Table 1$)$. The cut-off

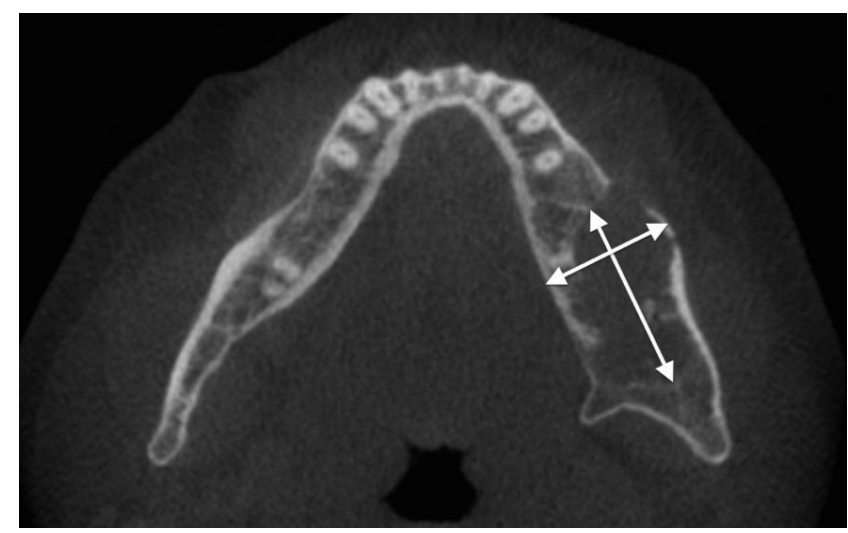

Fig. 1. Ameloblastoma. The width-to-length ratio is determined by measuring the ratio between the greatest buccolingual dimension and the greatest perpendicular anteroposterior dimension of the lesion on the axial view. In this case, the ratio is $25.95 / 37.05=0.7$.

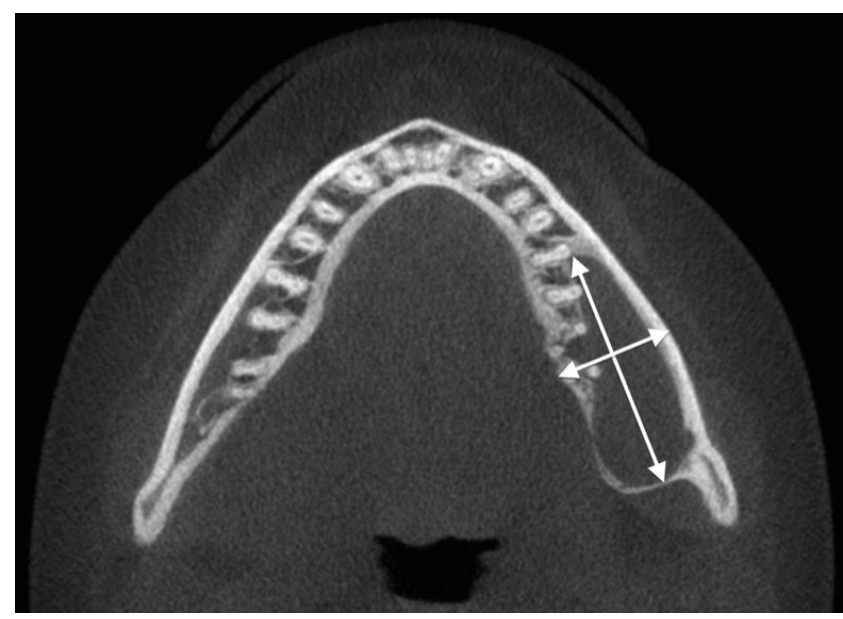

Fig. 2. Odontogenic keratocyst. The width-to-length ratio is $18.36 / 39.83=0.46$.

value with which the 2 types of lesions were differentiated was 0.5 . This value was determined by calculating the difference between the 5 th percentile of the value for ameloblastomas and the 75th percentile of the value for odontogenic keratocysts $(0.53-0.46=0.07)$ and dividing that difference by $2(0.07 / 2=0.03)$, assuming that the difference is exactly $50 \%$ for each group, then adding that to the 75 th percentile $(0.03+0.46=0.49)$.

\section{Discussion}

In the new fourth edition of the World Health Organization classification of head and neck tumors, keratocystic odontogenic tumor was reclassified back into the cystic category as odontogenic keratocyst. ${ }^{8}$ These lesions account 
Table 1. One-way ANOVA comparison of the width-to-length ratio values between odontogenic keratocyst and ameloblastoma

\begin{tabular}{lccccrr}
\hline \multicolumn{1}{c}{ Lesion } & Number & Mean & Standard deviation & Lower 95\% & Upper 95\% & $P$ value \\
\hline Ameloblastoma & 9 & 0.64 & 0.02 & 0.59 & 0.69 \\
Odontogenic keratocyst & 9 & 0.41 & 0.02 & 0.37 & 0.46 \\
\hline
\end{tabular}

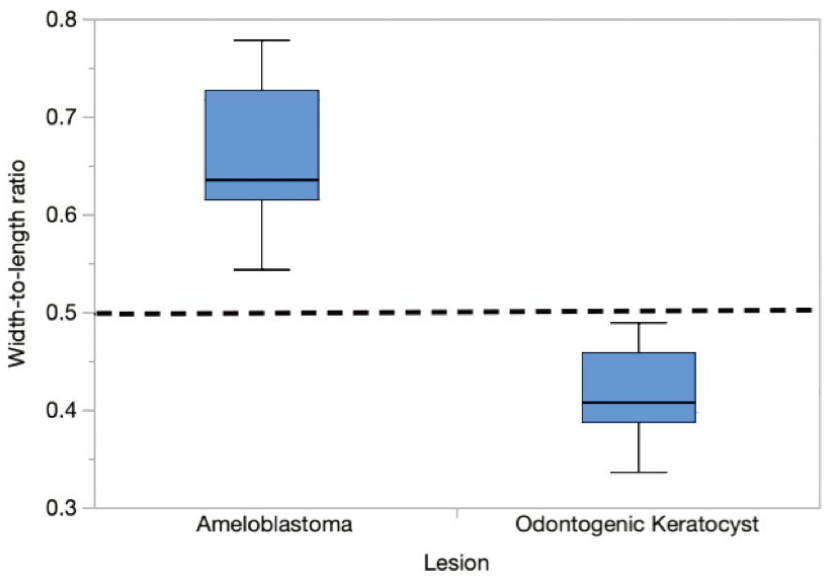

Fig. 3. Scatter plot comparing the width-to-length ratio between ameloblastomas $(n=9)$ and odontogenic keratocysts $(n=9)$. The dashed line represents the cut-off value of 0.5 .

for about $12 \%$ of all jaw cysts. ${ }^{2}$ Although odontogenic keratocysts occur in patients across a wide age range, they are discovered most frequently in patients in the second and third decades of life. Most studies have shown a slight male predilection. The lesions develop in the mandible twice as often as in the maxilla and most often in the posterior regions of both jaws. ${ }^{9}$ This cyst shows a propensity for recurrence, which has been reported in up to $30 \%$ of patients. ${ }^{10}$

Ameloblastoma is the most common odontogenic tumor of the jaw, representing $10 \%$ of all odontogenic tumors. ${ }^{1}$ It is characterized by aggressive, but benign growth. About $91 \%$ of ameloblastomas occur in the mandible, usually in the posterior region. Most lesions arise in the third to seventh decades of life, without a significant sex predilection. ${ }^{11}$

Ameloblastoma and odontogenic keratocyst may manifest as a unilocular or multilocular expansile mass, but the latter does not expand bone or resorb teeth to the same degree as the former. ${ }^{9,12}$ Odontogenic keratocyst tends to grow along the length of the bone with less bone expansion than expected, especially within the body of the mandible. The pattern of expansion of odontogenic keratocyst is similar to the fusiform expansion observed with fibrous dysplasia; in other words, the expansion appears to affect the bone more evenly, rather than the more concentric expan- sion seen with ameloblastoma. Although considerable expansion can occur and is more common with larger lesions, this expansion is not as impressive as that of ameloblastoma when the anteroposterior extent of the lesion is taken into account. ${ }^{9}$ In contrast, maxillary lesions more often cause gross balloon-like expansion with thinning and destruction of the overlying cortices. ${ }^{13}$ The more aggressive growth pattern in the maxilla than in the mandible may be explained by the flimsiness of the maxillary cortex, richer blood supply in the maxilla, and proximity to the sinonasal cavity and skull base.

Buccolingual expansion was reported in $62 \%$ and $91 \%$ of odontogenic keratocysts and ameloblastomas, respectively. ${ }^{9,12}$ No statistically significant difference was found between unicystic and solid/multicystic variants of ameloblastoma for the presence of expansion. ${ }^{12}$

The present study has shown that the width-to-length ratio, a quantitative measurement, is highly valuable for differentiating between ameloblastomas and odontogenic keratocysts in the body of the mandible. The width-to-length ratio is defined as the ratio between the greatest buccolingual dimension and the greatest perpendicular anteroposterior dimension of the lesion. To the best of our knowledge, this is the first study comparing the width-to-length ratio between ameloblastomas and odontogenic keratocysts. When the width-to-length ratio was used to differentiate between odontogenic keratocysts and ameloblastomas, the cut-off value with which the 2 lesions were differentiated was 0.5 . Presumably, it can be stated that lesions with a width-to-length ratio less than 0.5 (i.e., lesions for which the buccolingual dimension is $<50 \%$ of the anteroposterior dimension) are most likely odontogenic keratocysts, whereas those with a width-to-length ratio greater than 0.5 are probably ameloblastomas. This finding has not been described in previous studies.

Ariji et al. calculated the ratio of the buccolingual width on the affected side to that on the opposite side. They showed a significant difference in the degree of bone expansion between ameloblastomas and odontogenic keratocysts. ${ }^{4}$ Meng et al. calculated the "expansion ratio" by measuring the ratio between the bulging height and the length of the expanded cortex of the lesions in the maxilla. 
However, the value of buccal expansion was less significant for the differentiation of the maxillary lesions than for their mandibular counterparts. ${ }^{6}$

The width-to-length ratio may not help for small ameloblastomas or odontogenic keratocysts; however, such lesions are often asymptomatic and are discovered on routine radiographs when they attain a large size or when expansion is clinically evident.

Although this study is limited by its small sample size, the results are promising and suggest that further larger-scale studies are warranted. This work will add to a growing body of literature suggesting that various imaging features can significantly contribute to a correct diagnosis.

Given that ameloblastomas are resected with a wide margin and odontogenic keratocysts are usually marsupialized and/or enucleated, differentiation between these 2 conditions is imperative and lesions should always be submitted for a histologic examination.

In conclusion, this study demonstrated that the widthto-length ratio of ameloblastomas was significantly higher than that of odontogenic keratocysts. A cut-off value of 0.5 may differentiate odontogenic keratocysts from ameloblastomas. Further studies with a larger sample size are required to validate the role of the width-to-length ratio in the diagnostic imaging of jaw lesions.

\section{Acknowledgements}

The authors acknowledge Marcia Rojas Ramirez, DDS, MS, MPH (assistant professor, University of Kentucky College of Dentistry) for her professional assistance with statistical analysis.

\section{Conflicts of Interest: None}

\section{References}

1. Petrovic ID, Migliacci J, Ganly I, Patel S, Xu B, Ghossein R, et al. Ameloblastomas of the mandible and maxilla. Ear Nose Throat J 2018; 97: E26-32.
2. Johnson NR, Gannon OM, Savage NW, Batstone MD. Frequency of odontogenic cysts and tumors: a systematic review. J Investig Clin Dent 2014; 5: 9-14.

3. Kitisubkanchana J, Reduwan NH, Poomsawat S, Pornprasertsuk-Damrongsri S, Wongchuensoontorn C. Odontogenic keratocyst and ameloblastoma: radiographic evaluation. Oral Radiol (in press).

4. Ariji Y, Morita M, Katsumata A, Sugita Y, Naitoh M, Goto M, et al. Imaging features contributing to the diagnosis of ameloblastomas and keratocystic odontogenic tumours: logistic regression analysis. Dentomaxillofac Radiol 2011; 40: 133-40.

5. Alves DB, Tuji FM, Alves FA, Rocha AC, Santos-Silva AR, Vargas PA, et al. Evaluation of mandibular odontogenic keratocyst and ameloblastoma by panoramic radiograph and computed tomography. Dentomaxillofac Radiol 2018; 47: 20170288.

6. Meng Y, Zhao YN, Zhang YQ, Liu DG, Gao Y. Three-dimensional radiographic features of ameloblastoma and cystic lesions in the maxilla. Dentomaxillofac Radiol 2019; 48: 20190066.

7. Crusoé-Rebello I, Oliveira C, Campos PS, Azevedo RA, dos Santos JN. Assessment of computerized tomography density patterns of ameloblastomas and keratocystic odontogenic tumours. Oral Surg Oral Med Oral Pathol Oral Radiol Endod 2009; 108: 604-8.

8. Takata T, Slootweg PJ. Odontogenic and maxillofacial bone tumors. In: El-Naggar AE, Chan JK, Rubin Grandis J, Takata T, Slootweg PJ. WHO classification of head and neck tumours. 4th ed. Lyon, France: IARC; 2017. p. 203-60.

9. MacDonald-Jankowski DS. Keratocystic odontogenic tumour: systematic review. Dentomaxillofac Radiol 2011; 40: 1-23.

10. Johnson NR, Batstone MD, Savage NW. Management and recurrence of keratocystic odontogenic tumor: a systematic review. Oral Surg Oral Med Oral Pathol Oral Radiol 2013; 116 : e271-6.

11. MacDonald-Jankowski DS, Yeung R, Lee KM, Li TK. Ameloblastoma in the Hong Kong Chinese. Part 1: systematic review and clinical presentation. Dentomaxillofac Radiol 2004; 33: 71-82.

12. MacDonald-Jankowski DS, Yeung R, Lee KM, Li TK. Ameloblastoma in the Hong Kong Chinese. Part 2: systematic review and radiological presentation. Dentomaxillofac Radiol 2004; 33: 141-51.

13. Borghesi A, Nardi C, Giannitto C, Tironi A, Maroldi R, Di Bartolomeo $\mathrm{F}$, et al. Odontogenic keratocyst: imaging features of a benign lesion with an aggressive behaviour. Insights Imaging 2018; 9: 883-97. 